Document downloaded from:

http://hdl.handle.net/10251/171135

This paper must be cited as:

Soriano, A.; Sánchez, F.; Carrilero, V.; Pardo, A.; Vidal San Sebastian, LF.; Vazquez, C.;

Barbera, J.... (2013). Performance Evaluation of the Dual Ring MAMMI breast PET. IEEE. 14. https://doi.org/10.1109/NSSMIC.2013.6829103

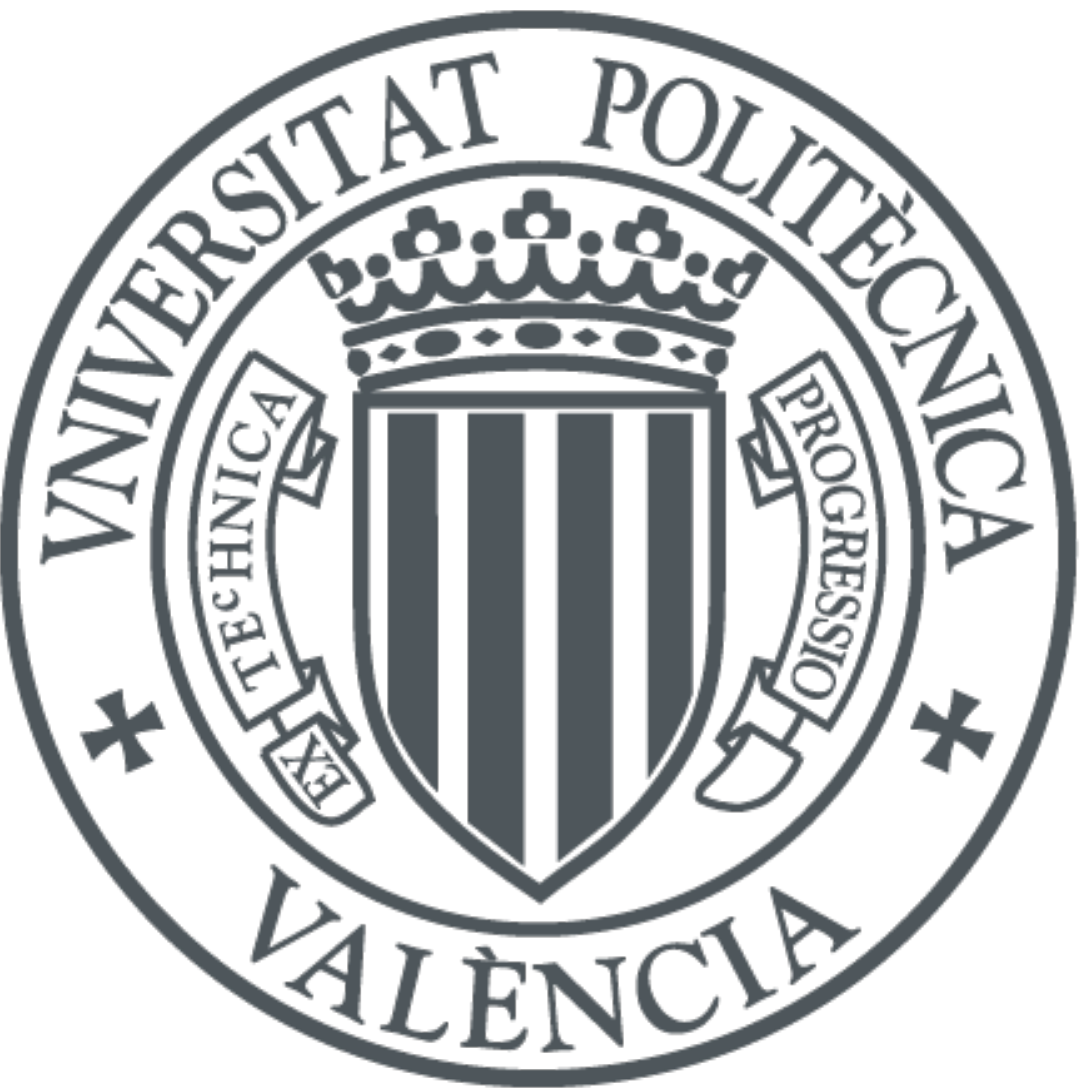

The final publication is available at

https://doi.org/10.1109/NSSMIC.2013.6829103

Copyright IEEE

Additional Information 


\title{
Performance evaluation of the Dual Ring MAMMI breast PET
}

\author{
A Soriano, F. Sánchez, V. Carrilero, A. Pardo, L.F. Vidal, C. Vázquez, J. Barberá, M. Seimetz, \\ M.J. Rodríguez-Alvarez, J.P. Rigla, L. Moliner, A. Iborra, L. Hernández, A.J. González, E. Crespo, P. Conde, \\ P. Bellido, L. Caballero, J.M. Benlloch
}

\begin{abstract}
MAMMI is a dedicated breast positron emission tomograph (PET) based on monolythic LYSO crystals, with a transaxial field of view (FOV) of $170 \mathrm{~mm}$. It has been upgraded by adding a second ring of detectors that extends the axial FOV from $40 \mathrm{~mm}$ to $94.4 \mathrm{~mm}$, in order to improve its sensitivity and reduce the acquisition time. In this work we present the performance evaluation of the dual ring MAMMI breast PET and a discussion about the contribution of the addition of a second ring of detectors, the compensation of the detector blur and the increase of the scintillator thickness.

Experimental measurements suggested on NEMA NU 4-2008 and NEMA NU 2-2007 have been conveniently adapted to the dimensions of the MAMMI. The addition of the second ring of detectors leads to a rise of the sensitivity from $1.8 \%$ to $3.6 \%$. The spatial resolution at one-fourth of the axial FOV $(1.5 \mathrm{~mm}$ axial, $1.6 \mathrm{~mm}$ tangential, $1.7 \mathrm{~mm}$ radial) is slightly better than that measured at the axial center $(1.9 \mathrm{~mm}$ axial, $1.8 \mathrm{~mm}$ tangential and radial), because of the $14 \mathrm{~mm}$ gap in between detection rings. The results obtained after the evaluation reflect a substantial performance improvement, specially in the absolute sensitivity, because of the changes introduced in the MAMMI PET.
\end{abstract}

Index Terms-Mammography, breast PET.

\section{INTRODUCTION}

Positron emission tomography (PET) has proven to have better sensitivity and specificity in detecting tumor lesions than $\mathrm{x}$-ray mammography. Detectability rates as high as $92 \%$ are achieved with PET in tumors larger than $2 \mathrm{~cm}$. But whole body PET are not effective for local staging of the breast because their efficiency declines in detecting small lesions $(<1 \mathrm{~cm})$ [1]. Dedicated PETs are based on high resolution detectors placed close to the breast. They permit to circumvent such a limitation and can be used in assessing therapy response, staging and restaging of breast cancer. This was the motivation for the development of the MAMMI (MAMmography with Molecular Imaging) dedicated breast PET [2].

The MAMMI PET described in [2] has an axial field of view (FOV) of $40 \mathrm{~mm}$ that translates axially to cover up to $170 \mathrm{~mm}$. However, full imaging of large breasts can require

Manuscript received November 4, 2007. This work was supported in part by the Spanish Plan Nacional de Investigación Científica, Desarrollo e Innovación Tecnológica (I+D+I) under Grant No. FIS2010-21216-CO2-01 and Valencian Local Government under Grants PROMETEOII/2013/010 and ISIC 2011/013.

A Soriano, F. Sánchez, L.F. Vidal, M. Seimetz, M.J. Rodríguez-Alvarez, J.P. Rigla, L. Moliner, A. Iborra, L. Hernández, A.J. González, E. Crespo, P. Conde, P. Bellido and J.M. Benlloch are with the Institute of Instrumentation for Molecular Imaging, CSIC - UPV - CIEMAT. C/ Camino de Vera s/n, 46022 Valencia (SPAIN) e-mail: asoriano@i3m.upv.es

V. Carrilero, A. Pardo, C. Vázquez, J. Barberá and L. Caballero are with Oncovision. C/ Eduardo Primo Yúfera, 3. 46013 Valencia (SPAIN) several acquisitions in step and shoot mode, that can last more than 20 min. An upgrade of the MAMMI PET has been proposed in order to cut down acquisition times. It consists in the addition of a second ring of detectors. The thickness of the scintillator has been increased from $10 \mathrm{~mm}$ to $12 \mathrm{~mm}$ in order to enhance the efficiency in detection of photons. An improved model of the scanner, accounting for the photon penetration into the scintillator [3], has been considered in the iterative image reconstruction.

In this work we have evaluated the performance of the dual ring configuration of the MAMMI PET based on the measurements and procedures described in NEMA NU 2-2007 [4] and NEMA NU 4-2008 protocols [5]. The performance of the dual ring configuration has been compared with that of the single ring configuration presented in [2]. A concise description of the MAMMI dedicated breast PET and the measurements conducted in this work is given in Sec. II. The performance of the dual ring configuration of the MAMMI PET are summarized in Sec. III. Finally, in Sec. IV are discussed the major improvements achieved with the dual ring configuration of the MAMMI PET.

\section{MATERIALS AND METHODS}

\section{A. MAMMI breast PET}

The MAMMI dedicated breast PET [2] has a full ring geometry forming a dodecagon with an aperture of $186 \mathrm{~mm}$. The scanner has been designed so that the patient lies down in prone position. Each detector module consists of a single monolithic LYSO crystal $(10 \mathrm{~mm}$ thick), with a trapezoidal shape $\left(40 \times 40 \mathrm{~mm}^{2}\right.$ entrance face and $50 \times 50 \mathrm{~mm}^{2}$ exit face) that minimizes dead areas in between detectors. The scintillator is optically coupled to a H8500 position sensitive photomultiplier (PSPMT) from Hamamatsu Photonics, and an Anger resistor network is used to measure the impact position and energy deposited. The transaxial FOV has a diameter of $170 \mathrm{~mm}$ and the axial FOV is $40 \mathrm{~mm}$. The detection ring translates axially extending the axial FOV up to $170 \mathrm{~mm}$, in order to image the whole breast.

\section{B. Dual Ring configuration of the MAMMI breast PET}

Several modifications have been proposed to upgrade the MAMMI in order to improve its sensitivity and thus reduce the total acquisition time and the dose injected to the patient.

- Scintillator thickness increase: from $10 \mathrm{~mm}$ to $12 \mathrm{~mm}$, in order to enhance the efficiency in the detection of the 
$511 \mathrm{keV}$ photons produced after the annihilation of a positron.

- Second ring of detectors: in order to extend the axial size of the FOV from $40 \mathrm{~mm}$ to $94.4 \mathrm{~mm}$. This reduces the number of frames required to image the whole breast, thus reducing the total acquisition. The addition of a second ring also increases the solid angle covered by the detector which leads to increase the sensitivity of the scanner.

- Modelling of photon penetration depth: contributes to minimize the parallax error [3] and circumvents the detriment in spatial resolution caused by large acceptance angles in thick scintillators. The scintillator is discretized into volumetric bins, and we calculate the likelihood of an event occurred in a given LOR being registered in a pair of volumetric bins. This information is then incorporated as a blur term into the detection probability matrix $(P)$ employed in the maximum likelihood expectation maximization (MLEM) image reconstruction.

$$
P=P_{\text {sens }} \mathbf{P}_{\text {blur }} P_{\text {att }} P_{\text {geom }} P_{\text {positron }}
$$

where each term accounts for the different physical interactions involved in the measurement: positron range $\left(P_{\text {positron }}\right)$, geometrical efficiency and photon noncollinearity $\left(P_{\text {geom }}\right)$, photon attenuation in the body $\left(P_{a t t}\right)$, photon attenuation and Compton scattering in the scintillator $\left(P_{\text {blur }}\right)$ and sensitivity differences among detectors $\left(P_{\text {sens }}\right)[6]$.

\section{Experimental Measurements}

The phantoms, sources and measurements proposed in NEMA standards [4], [5] were conveniently adapted to evaluate the performance of the scanner, since the characteristics of dedicated breast PETs do not exactly suit none of them. The experimental measurements were done following the same procedure as in [2] so that we were able to compare the results with those achieved with the MAMMI breast PET with one ring of detectors.

The sensitivity was measured with a $1 \mathrm{~mm}$ in diameter ${ }^{22} \mathrm{Na}$ source with an activity of $370 \mathrm{kBq}$, that was moved along the axis of the scanner in step sizes of $4 \mathrm{~mm}$.

The spatial resolution was measured with a $0.25 \mathrm{~mm}$ in diameter ${ }^{22} \mathrm{Na}$ point-like source placed at the center and at one-fourth of the axial FOV. Acquisitions were done at radial distances from the center of the scanner up to $70 \mathrm{~mm}$ at increments of $5 \mathrm{~mm}$. The full width at half maximum (FWHM) of the reconstructed point source at all three directions has been measured, the effect of the finite size of the source has not been considered in the reported values.

The noise equivalent counts (NEC) were evaluated with a high density polyethylene cylinder $225 \mathrm{~mm}$ long and $70 \mathrm{~mm}$ in diameter, with a hole of $3.2 \mathrm{~mm}$ drilled at $13 \mathrm{~mm}$ of the center where a tube with a ${ }^{18} \mathrm{~F}$ solution was placed. The phantom has been extended from $170 \mathrm{~mm}$ to $225 \mathrm{~mm}$ in order to account for the activity outside the FOV. Measurements every 12 min have been conducted in order to extract the prompt, trues, scatter, random and NEC curves as the activity inside the FOV varies, according to the protocol described in [5].

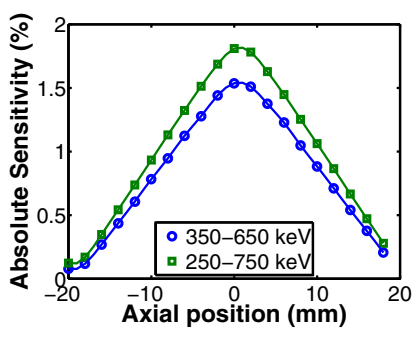

(a)

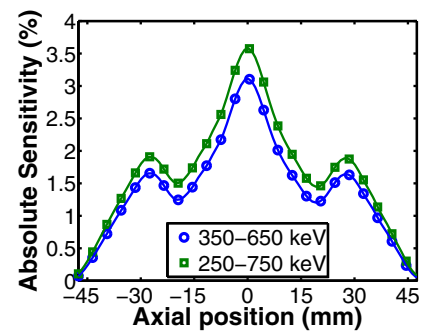

(b)
Fig. 1. Comparison of sensitivity measurements with (a) one ring [2] and (b) two rings of detectors.

\section{RESULTS}

\section{A. Sensitivity}

The addition of the second ring of detectors leads to an increase of the sensitivity of the scanner (see Fig. 1). The axial position of the point source is referenced to the center of the FOV in both single (Fig. 1(a)) and dual ring (Fig. 1(b)) configurations of the MAMMI PET. A maximum sensitivity of $3.6 \%$ is reached at the center of the scanner when considering a $(250 \mathrm{keV}-750 \mathrm{keV})$ energy window and $3.1 \%$ if the energy window is enlarged up to $(350 \mathrm{keV}-650 \mathrm{keV})$, typically defined in clinical studies. These values almost double those measured with the one ring configuration of the MAMMI PET [2].

The two local maxima appreciated in Fig. 1(b) coincide with the centers of each of the detection rings. The sensitivity achieved at these two local maxima are slightly better than that measured in [2] with one detection ring. Measurements from only one of the rings have been considered in order to estimate the contribution of the increase of the scintillator thickness from $10 \mathrm{~mm}$ to $12 \mathrm{~mm}$ to the scanner's sensitivity. An improvement in sensitivity of $7 \%$ has been measured when considering a scintillator $12 \mathrm{~mm}$ thick which is smaller than the improvement of $25 \%$ in sensitivity predicted by theory. This result suggests that the improvement in sensitivity observed in the dual ring configuration is mainly due to the increase of the solid angle covered by the detector.

\section{B. Spatial Resolution}

The comparison of the spatial resolution measured with the single and dual ring configurations of the MAMMI PET are compared in Fig. 2. $1.9 \mathrm{~mm}$ axial and $1.8 \mathrm{~mm}$ radial and tangential spatial resolution values have been measured in the center of the dual ring MAMMI PET, which are similar to the values $1.6 \mathrm{~mm}, 1.8 \mathrm{~mm}$ and $1.9 \mathrm{~mm}$ measured along axial, radial and tangential directions with one ring of detectors [2]. Despite similar values are obtained in the center of the scanner, an improvement in spatial resolution is appreciated in the measurements with the dual ring configuration of the MAMMI PET, specially as the point-like source is moved away from the axis of the scanner.

It has to be pointed out that the spatial resolution measurements at one-fourth of the axial FOV (Fig. 2(d)) are better than those conducted at the center of the FOV (Fig. 2(b)). Spatial resolutions of $1.5 \mathrm{~mm}, 1.6 \mathrm{~mm}$ and $1.7 \mathrm{~mm}$ along 


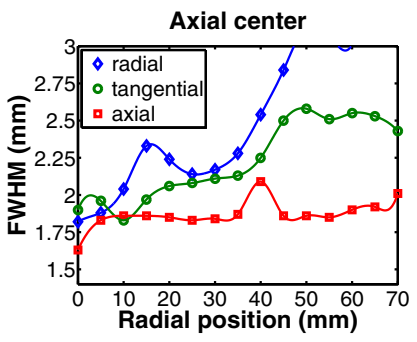

(a)

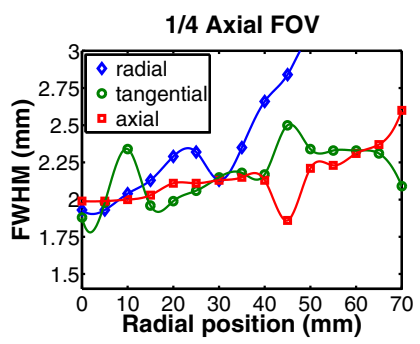

(c)

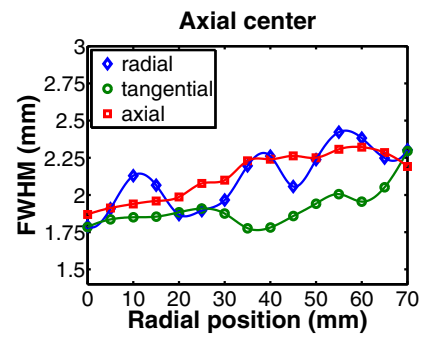

(b)

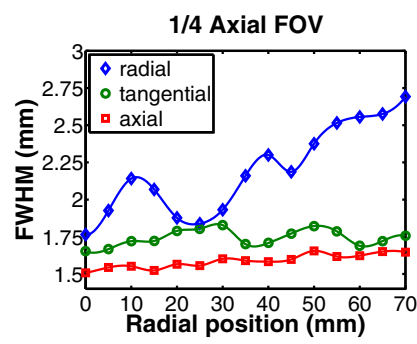

(d)
Fig. 2. Comparison of spatial resolution measurements in the center of the scanner with (a) one ring and (b) two rings of detectors, and at one-forth of the axial FOV with (c) one ring and (d) two rings of detectors.

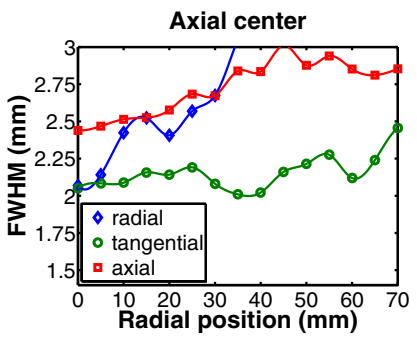

(a)

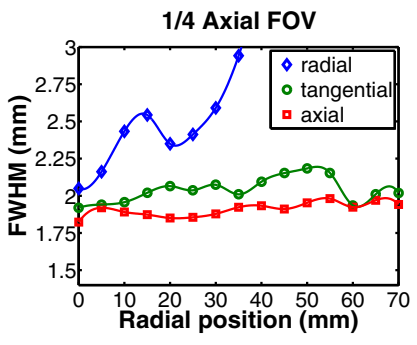

(b)
Fig. 3. Measurements of spatial resolution with the dual ring configuration of the MAMMI PET (a) in the center and (b) at one-fourth of the axial FOV when the photon penetration is not accounted for in the reconstruction model.

axial, radial and tangential directions have been measured in the center at one-fourth of the axial FOV with the dual ring configuration of MAMMI PET. This behavior is due to the fact that the positions at one-fourth of the axial FOV coincide with the center of each ring of detectors, while the axial center of the scanner correspond to a small $14.4 \mathrm{~mm}$ gap in between detection rings. The fall of the density of lines of response in this region leads to a slight degradation of the spatial resolution measured in the center of the scanner. The spatial resolution values along axial and tangential directions are kept below $1.75 \mathrm{~mm}$ in most of the FOV (Fig. 2(d)). Only the radial FWHM of the point-like source takes values above $2.5 \mathrm{~mm}$ in the outer region of the FOV (distance $>50 \mathrm{~mm}$ ). In any case, better spatial resolution values are obtained with the dual ring configuration of the MAMMI PET, than those shown in [2] for the MAMMI PET with one ring of detectors.

In order to quantify the contribution of the penetration of photons into the scintillator to the measurement of the spatial resolution [3], the acquisitions of the point-like source have been reconstructed without accounting for the penetration of
TABLE I

COMPARISON OF SCATTER FRACTION (\%) NEC PEAK (KCPS @ MBQ) MEASURED WITH THE SINGLE AND DUAL RING MAMMI PET.

\begin{tabular}{clcc}
\hline \hline \multicolumn{2}{c}{ Energy (keV) } & 350 - 650 & $\mathbf{2 5 0}-\mathbf{7 5 0}$ \\
\hline \multirow{2}{*}{ Single Ring } & SF (\%) & 18.2 & 20.8 \\
& NEC (kcps @ MBq) & $18 @ 38$ & $25 @ 44$ \\
\hline \multirow{2}{*}{ Dual Ring } & SF (\%) & 30.8 & 44 \\
& NEC (kcps @ MBq) & $34 @ 27$ & $34 @ 27$ \\
\hline
\end{tabular}

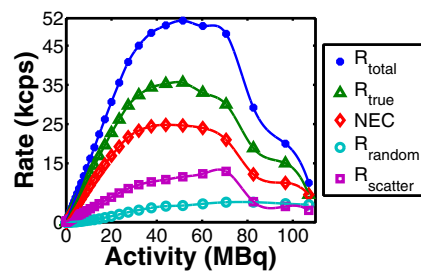

(a)

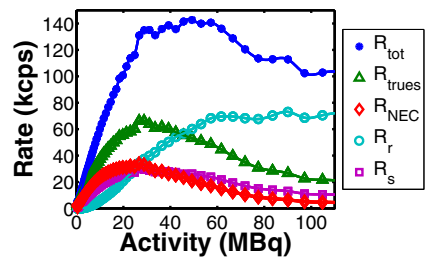

(b)
Fig. 4. Comparison of NEC performance with (a) one ring [2] and (b) two rings of detectors.

photons and the results are shown in Fig. 3. Comparable spatial resolution values have been measured in Fig. 3(b) and Fig. 2(a) since both scenarios correspond to locations of the point-like source along the center of one of the rings of detectors. The comparison of Fig. 2(b) with Fig. 3(a) and Fig. 2(d) with Fig. 3(b) reflects the contribution of the considerations of the penetration of photons into the spatial resolution.

\section{Noise Equivalent Counts (NEC)}

The increase of the axial FOV from $40 \mathrm{~mm}$ to $94.4 \mathrm{~mm}$ because of the addition of a second ring of detectors rises the likelihood of detection of scatter events. This enlargement of the FOV leads to an increase of the scatter fraction (SF) from $18 \%$ to $31 \%$ when the $(350 \mathrm{keV}-650 \mathrm{keV})$ energy window was chosen. As it is appreciated in Table I, if the $(250 \mathrm{keV}$ $750 \mathrm{keV}$ ) energy window is fixed the rise of SF is even more pronounced since the widening of the energy window only favors the detection of scattered events.

The comparison of Fig. 4(a) and Fig. 4(b) shows up that the rate of prompts detected in the dual configuration of the MAMMI PET doubles that measured for single ring configuration because of the increase in sensitivity. This causes that the NEC peak raises from $18 \mathrm{kcps}$ [2] to $34 \mathrm{kcps}$ in this work when the $(350 \mathrm{keV}-650 \mathrm{keV})$ energy window is considered. The strong increase of the SF with the energy window constrains the improvement of the NEC peak when the energy window is opened up to $(250 \mathrm{keV}-750 \mathrm{keV})$. The higher amount of prompt events detected with the dual ring configuration of the MAMMI PET in comparison with the single ring configuration is one of the reasons why the NEC peak is reached at $27 \mathrm{MBq}$ which is smaller than that obtained for the single ring configuration (44 MBq) [2].

\section{Discussion}

The dual ring configuration of the MAMMI PET has an axial FOV of $94.4 \mathrm{~mm}$, which allows to decrease the number of frames required to scan the whole breast. The acquisition time 
per frame can also be reduced since the sensitivity measured with the dual ring MAMMI PET $(3.6 \%)$ is twice of that measured with one ring of detectors $(1.8 \%)$. The improvement in sensitivity is mainly due to the solid angle covered by the dual ring MAMMI PET. The improvement in sensitivity because of the increase of the scintillator thickness is smaller than predicted by theory. The spatial resolution measured at $1 / 4$ of the axial FOV $(1.5 \mathrm{~mm})$ is better than that measured in the center $(1.9 \mathrm{~mm})$, because of the $14.4 \mathrm{~mm}$ air gap in between rings. The consideration of the penetration of photons into the scintillator compensates the worsening of the spatial resolution in the central region of the FOV.

As expected, the increase of the sensitive area leads to a rise of the scatter fraction. The $34 \mathrm{kcps}$ NEC peak achieved with the dual ring configuration almost doubles the (18 kcps) NEC peak measured with one ring of detectors when the $(350 \mathrm{keV}$ $-650 \mathrm{keV}$ ) energy window is taken into account.

The improvement in sensitivity in the dual ring configuration of the MAMMI PET leads to a substantial increase in the number of counts registered at a given activity, and therefore the NEC peak is reached at an activity of $27 \mathrm{MBq}$ while it was achieved at $44 \mathrm{MBq}$ with the single ring configuration of the MAMMI PET. Despite typical doses in clinical practice are below these limits, several improvements in the readout electronics are foreseen in order to further increase the activity at which the NEC peak is reached.

\section{CONCLUSION}

The upgrade with a second ring of detector modules permits to double the sensitivity of the MAMMI dedicated breast PET. The increase of the detection area and the solid angle covered by the detector is the major cause of the increase in sensitivity observed. The improvement of the iterative reconstruction by modeling the photon penetration into the scintillator permits to increase the acceptance angle of impinging photons in the detector while improving the spatial resolution. Better spatial resolution values have been measured at $1 / 4$ of the axial FOV than in the center of the FOV because the position at $1 / 4$ of the axial FOV coincides with the center of one of the scanner's detection rings. The addition of a second ring of detectors also contributes to enhance the NEC peak values. The rise of the count rate because of the enhancement in sensitivity is one of the reasons for the decrease in the activity at the NEC peak. Despite typical doses in the breast in clinical practice imply activities below $10 \mathrm{MBq}$ inside the MAMMI FOV, several readout improvements are foreseen to further improve the NEC curves.

\section{REFERENCES}

[1] N. Avril, C. Rosé, M. Schelling, J. Dose, W. Kuhn, S. Bense, W. Weber, S. Ziegler, H. Graeff, and M. Schwaiger, "Breast imaging with positron emission tomography and fluorine-18 fluorodeoxyglucose: Use and limitations," Journal of Clinical Oncology, vol. 18, no. 20, pp. 3495-3502, October 2000.

[2] L. Moliner, A. J. González, A. Soriano, F. Sánchez, C. Correcher, A. Orero, M. Carles, L. F. Vidal, L. Caballero, M. Seimetz, C. Vázquez, and J. M. Benlloch, "Design and evaluation of the mammi dedicated breast pet," Medical Physics, vol. 39, no. 9, pp. 5393-5404, September 2012.
[3] A. Soriano, A. J. González, F. Sánchez, P. Conde, L. Moliner, A. Orero, M. J. Rodríguez-Alvarez, L. F. Vidal, and J. M. Benlloch, "Minimization of parallax error in dedicated breast PET," IEEE Trans. on Nucl. Sci., vol. 60, no. 2, pp. 739-745, April 2013.

[4] "NEMA standards publication NU 2-2007: Performance measurements of positron emission tomographs," National Electrical Manufacturers Association, Tech. Rep., 2007.

[5] "NEMA standards publication NU 4-2008: Performance measurements of small animal positron emission tomographs," National Electrical Manufacturers Association, Tech. Rep., 2008.

[6] J. Qi, R. M. Leahy, S. R. Cherry, A. Chatziioannou, and T. Farquhar, "High-resolution 3D Bayesian image reconstruction using the microPET small-animal scanner," Physics in Medicine and Biology, vol. 43, pp. 1001-1013, 1998. 